\title{
STATUS OF HIGH INTENSITY POLARIZED ELECTRON GUN PROJECT
}

\section{E. Tsentalovich}

\author{
MIT - Bates
}

21 Manning Road, Middleton, MA,01949, USA

E-mail: evgeni@mit.edu

MIT-Bates in collaboration with BNL investigate the possibility of building a polarized electron gun with extremely high intensity. The design implements a separate preparation chamber, load lock, ring-shaped beam and active cathode cooling. Very good vacuum conditions have been achieved in both the gun chamber and the preparation chamber. Reliable cathode transfer between the load lock, the preparation chamber and the gun chamber has been demonstrated. The design of the beam line and the beam dump has been completed, and the beam line fabrication is under way.

XVth International Workshop on Polarized Sources, Targets, and Polarimetry September 9-13, 2013

Charlottesville, Virginia, USA 


\section{Introduction}

MIT-Bates investigates a possibility to build a very high intensity polarized electron gun to investigate techniques for increasing polarized electron intensities for an Electron-Ion Collider. This development is crucial for the eRHIC project [1], as noted in the 2007 NSAC Long Range Plan and the November 2009 report of the Electron-Ion-Collider Advisory Committee. eRHIC requires average polarized electron currents on the order of $50 \mathrm{~mA}$, whereas existing polarized electron sources typically operate in the 100-200 $\mu \mathrm{A}$ range, and have achieved average currents of about several $\mathrm{mA}$ with very short lifetime.

One of the reasons for a short life time is the fact that all existing guns have rather poor thermal connection to the cathode. A laser beam with a very significant laser power has to be used for the photo injection and it overheats the crystal.

However the most challenging problem for the lifetime is produced by ion back bombardment. Electron beam ionizes the molecules of the residuum gases in the cathode-anode gap, ions accelerate toward the cathode and damage it on the impact. Tremendous efforts have been applied to improve the vacuum inside the guns, but the ion bombardment remains the main limiting factor in gun intensity.

It was shown that the ion damage depends on the size of the active area of the photocathode. With large photocathodes the damage is spread over a large area and allows for the longer lifetime. It was also demonstrated [2,3] that the ions tend to damage the central area of the cathodes mostly due to the focusing properties of the anode.

A large area cathode will be used in the gun. The new feature of the installation will be annular shape of the beam. A ring-shaped laser beam will be formed with an axicon lens. The central area of the cathode, where most of the damage is concentrated will not be used at all. The active cathode cooling will be implemented.

\section{Gun Layout.}

The installation consists of three chambers: the gun chamber, the preparation chamber and the load-lock chamber /Fig.1/. The first two chambers must have very good vacuum, and they are not assumed to be vented ever. The new cathodes are placed into the load-lock chamber, and after this chamber is pumped out, the valve between the load-lock and the preparation chamber opens and the cathodes are transferred into the preparation chamber with a magnetic-coupled manipulator. Heat cleaning and activation takes place in the preparation chamber, and then the activated cathode is transferred into the gun chamber with a similar manipulator.

The GaAs crystal is mounted on a Molybdenum puck with a Tantalum cup pressing it to the puck. An indium foil is inserted between the puck and the crystal. During the first activation the foil melts and solders the crystal to the puck providing very good thermal connection.

The load-lock chamber is equipped with a rack that can hold up to four pucks. The rack could be moved in the vertical direction to place the desired puck against the fork of the manipulator.

The heart of the preparation chamber is a carousel that can be rotated and also be moved in vertical directions. The pucks are placed into the receptables on the wings of the carousel. The 
carousel moves the photocathodes to either heat-cleaning or activation station. There are two heat cleaning stations and two activation in the preparation chamber. Each heating station is equipped with a PBN heater, thermocouple for the reference measurements and a view port for the pyrometer. The activation station are equipped with cooling rods, Cesium dispensers, NF3 leak valve and windows for laser light. The cooling rods could be biased to apply negative voltage to the crystals.

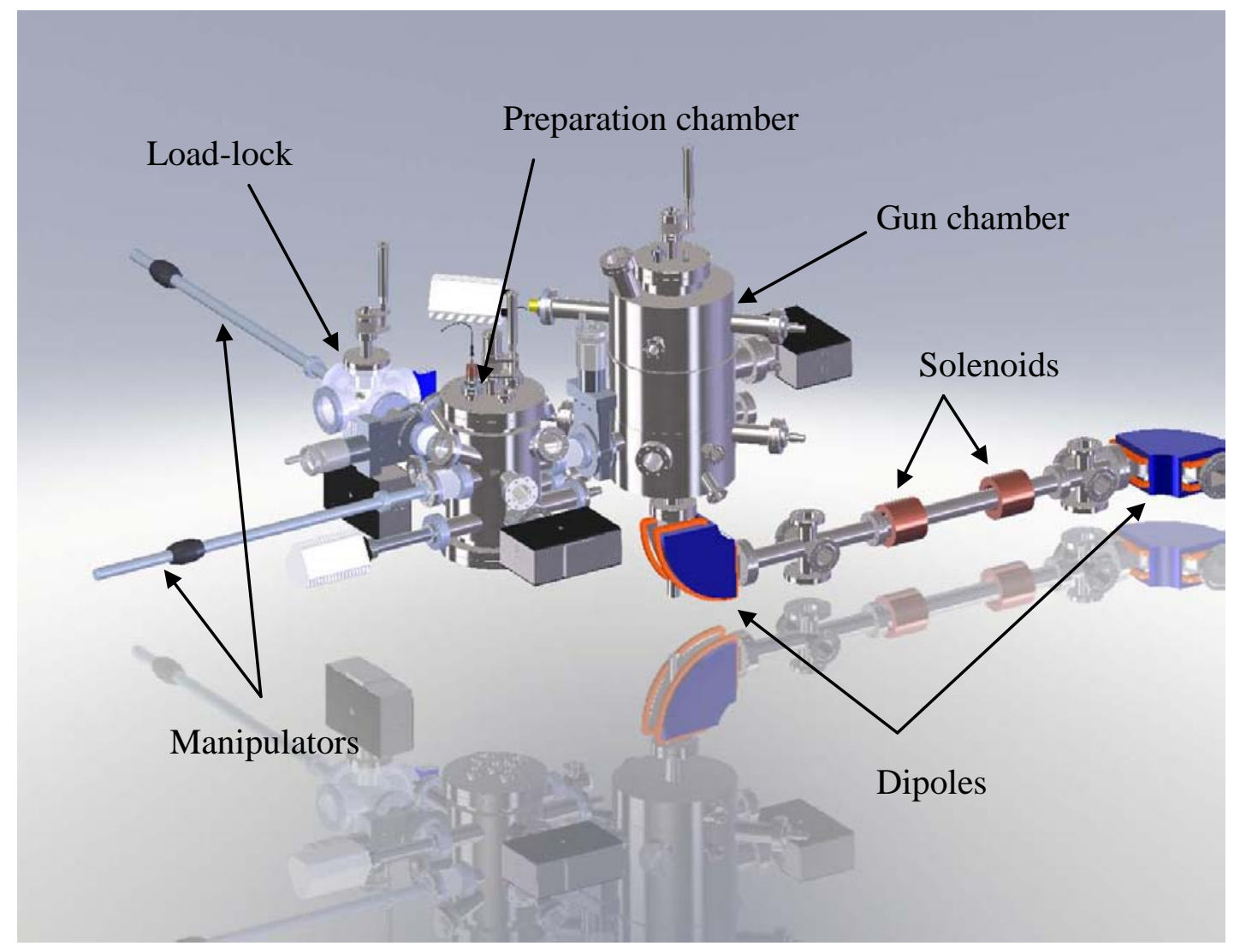

Figure 1. The gun and beam line layout.

The gun chamber is manufactured from a stainless steel. The cathode assembly is suspended on three long ceramic tubes. Two of these tubes serve as pipes to deliver cooling agent to and from the cathode. A Fluorinert is used as a cooling agent. This liquid has virtually zero conductivity and very good electrical strength. The third tube serves as a conduit for HV cable. The working voltage of the cathode is $120 \mathrm{kV}$. An additional ceramic rod attached to a Linear Transfer Mechanism (LTM) at the top of the chambers moves the cathode in vertical direction allowing to open a gap between the cathode and the heat exchanger and insert the puck with a crystal through the side port using a magnetic-coupled manipulator. Conical shape of the interface between the puck and heat exchanger insures self-centering and good thermal connection.

The cathode assembly is surrounded by a polished field shield to prevent a field emission. The gun was processed to $150 \mathrm{kV}$. After the processing there is no any signs of the activity (measurable dark current or vacuum excursions) at the working voltage of $120 \mathrm{kV}$.

The anode is disconnected from the ground potential and is biased to $1 \mathrm{kV}$ in order to reflect the ions produced outside the cathode-anode gap and trapped in the electron beam. 
All three chambers are equipped with multiple view ports for observation during the vacuum manipulation. The halogen bulbs installed in vacuum provide excelent illumination.

The beam line consists of the two $90^{\circ}$ dipole magnets, several focusing solenoids, steering coils and a beam dump. The beam propagation in the beam line was carefully modeled. It is extremely important to minimize the beam losses near the gun. At such high intensity even $10^{-6}$ losses could be fatal for the crystal. The simulations indicated that our losses will be much smaller than $10^{-6}$ up to the second dipole. A special attention was given to the "extreme" rays electrons produced at the very edge of the crystal. Although the simulation suggested that even these electrons will not hit the walls, it is unclear how accurately the calculations include the influence of the Tantalum cup which produces a tiny step on the flat surface. In order to minimize the risk produced by the "extreme" electrons, the outer $1 \mathrm{~mm}$ of the crystal is screened during the activation, so Quantum Efficiency of this area is very low.

\section{Status of the project.}

All three chambers have been assembled and baked. Very good vacuum has been achieved in the gun chamber, The pressure is less than $1 \cdot 10^{-11}$ torr, dominated by hydrogen. The vacuum in the preparation chamber is higher by about factor of 2 .

Very reliable vacuum manipulation has been demonstrated. The pucks with photo cathodes were moved between different chambers routinely.

The photocathodes have been heat cleaned and activated, and rather high Quantum Efficiency has been observed (about $2 \%$, for bulk GaAs crystals, with a light at $\lambda=804 \mathrm{~nm}$ ).

The beam line is currently under assembly.

\section{References}

[1] V.Ptitsyn, From HERA To Future Electron-Ion Colliders, Proc. of the Particle Accelerator Conference, p.1927-1931 (2007).

[2] J.Grames et al, Proc. of 12th Intern. PST Workshop, Upton, AIP Conf. Proc. 980, p.110 (2007)

[3] E.Tsentalovich, Proc. of 18th Spin Ph. Symp., Charlottesville, AIP Conf. Proc. 1149, p.997 (2009). 